\title{
SARS-COV-2 Infection in a Term Neonate Presenting with Respiratory Failure on Day 3 of Life
}

\author{
Kanya Mukhopadhyay ${ }^{1} \cdot$ Ashish Agarwal $^{2} \cdot$ Veena Laxmi $^{2} \cdot$ Gursimran Kaur Mohi $^{3} \cdot$ Lakshmi PVM $^{4}$
}

Received: 1 August 2020 / Accepted: 21 August 2020 / Published online: 26 August 2020

(C) Dr. K C Chaudhuri Foundation 2020

To the Editor: Symptomatic Corona virus disease-2019 (COVID-19) disease due to Severe acute respiratory syndrome coronavirus 2 (SARS-COV-2) infection in neonates is rare. Neonates may acquire infection either by vertical transmission from mother or by horizontal transmission. We report a case of an asymptomatic term neonate who suddenly deteriorated on day three and tested positive for SARS-COV-2 infection.

A $38 \mathrm{wk}, 3 \mathrm{~kg}$ female neonate was delivered by elective cesarean section due to placenta previa. Mother was not tested for COVID-19 before delivery. On day 2 the baby developed jaundice (serum bilirubin $14.7 \mathrm{mg} / \mathrm{dl}$ ). On day 3 she had sudden onset of paroxysms of cough after the feed and developed apnea. She was intubated immediately and after initial cardiopulmonary resuscitation, referred to our emergency on manual positive pressure ventilation. No symptoms were noticed by parents preceding this episode. There was no setting of early onset bacterial infection. At arrival she had severe bradycardia and no respiratory efforts. She could not be resuscitated and declared dead at $78 \mathrm{~h}$ of life. The post mortem nasopharyngeal swab for COVID-19 RT-PCR was positive and the ORF gene CT was 28 and RdRp gene CT was 21 (BGI kit). No $\mathrm{X}$-ray or blood test or autopsy could be done. COVID19 RT-PCR of parents, family contacts and health care workers tested negative.

Kanya Mukhopadhyay

kanyapgi@gmail.com

1 Neonatal Unit, Department of Pediatrics, Post Graduate Institute of Medical Education and Research, Chandigarh 160012, India

2 Department of Pediatrics, Post Graduate Institute of Medical Education and Research, Chandigarh, India

3 Department of Virology, Post Graduate Institute of Medical Education and Research, Chandigarh, India

4 Department of Community Medicine and School of Public Health, Post Graduate Institute of Medical Education and Research, Chandigarh, India
The source of infection in our case could not be traced; however some visitors and asymptomatic health care workers may not have been tested. Vertical transmission cannot be ruled out as mother was tested $8 \mathrm{~d}$ postpartum and she might have turned negative by this time or false-negative. Horizontal transmission from unknown sources cannot be ruled out.

Systematic reviews and meta-analyses in neonates show very low rate of infection (2.7\% to $3.6 \%$ ) who are mostly asymptomatic [1-3]. Vertical transmission is rare and cannot be proven without testing of amniotic fluid, cord blood and placenta. Vertical transmission can occur during vaginal delivery however, cesarian section does not reduce infection [4].

A recent similar case reported from Italy presented with severe hypoxemia on day 5 without overt signs of respiratory distress. The mother tested positive on day 2 postpartum and may be horizontal transmission [5].

Our baby was born in a basic level health care facility and early hypoxemia could have been missed. The possibility of a false positive report is very unlikely as there were severe respiratory symptoms and RT-PCR is highly specific with very low false positivity rate of $3 \%$ [6].

Thus, to conclude, during this ongoing COVID-19 pandemic, any neonate presenting with unexplained respiratory symptoms should be tested for SARS-COV-2 infection.

\section{Compliance with Ethical Standards}

Conflict of Interest None.

\section{References}

1. Gatzinger F, Garcia BS, Julian AN, et al. COVID- 19 children and adolescents in Europe: a multinational and multicountry cohort study. Lancet. 2020;4:653-61. https://doi.org/10.1016/S23524642(20)30177-2.

2. Yang Z, Liu Y. Vertical transmission of severe acute respiratory syndrome coronavirus 2: a systematic review. Am J Perinatol. 2020;37:1055-60. https://doi.org/10.1055/s-0040-1712161. 
3. Goh XL, Low YF, Ng CH, Amin Z, Ng YPM. Incidence of SARSCOV-2 vertical transmission : a meta analysis. Arch Dis Child Fetal Neonatal Ed. 2020. https://doi.org/10.1136/archdischild-2020319791.

4. Walker KF, O'Donoghue K, Grace N, et al. Maternal transmission of SARS-COV-2 to the neonate, and possible routes for such transmission: a systematic review and critical analysis. BJOG. 2020. https:// doi.org/10.1111/1471-0528.16362.

5. Sinelli M, Paterlini G, Citterio M, Marco AD, Fedeli T, Ventura ML. Early neonatal SARS-CoV-2 infection manifesting with hypoxemia requiring respiratory support. Pediatrics. 2020;146:e20201121. https://doi.org/10.1542/peds.2020-1121.

6. Cohen AN, Kessel B. False positives in reverse transcription PCR testing for SARS-CoV-2. medRxiv. 2020. https://doi.org/10.1101/ 2020.04.26.20080911.

Publisher's Note Springer Nature remains neutral with regard to jurisdictional claims in published maps and institutional affiliations. 